\title{
Osmotic Pressure of Semidilute Solutions of Branched Polymers
}

\author{
Yuji Higo, Noboru UENO, and Ichiro NODA \\ Department of Synthetic Chemistry, Faculty of Engineering, \\ Nagoya University, Furo-cho, Chikusa-ku, Nagoya 464, Japan
}

(Received December 3, 1982)

\begin{abstract}
Osmotic pressures for branched polymers in a good solvent were measured over a wide range of concentrations. Two types of branched polymers, star-shaped poly ( $\alpha$-methylstyrene)s having three branches and comb-shaped polystyrenes having 15-16 branches, were studied. The data were analyzed using the scaling law. The difference in the reduced osmotic pressure between star-shaped polymers having three branches and the corresponding linear polymers results from the difference in radii of gyration. In branched polymers having many branches, there is a region from which the segments of other molecules are excluded as a result of high segment density.

KEY WORDS Osmotic Pressure / Semidilute Solution / Branched Polymer /

Star-Shaped Polymer / Comb-Shaped Polymer / Scaling Law /
\end{abstract}

The osmotic pressure of linear polymers in good solvents shows different concentration dependence in three different regions, i.e., dilute $\left(C<C^{*}\right)$, semidilute $\left(C>C^{*}\right)$, and concentrated solutions. ${ }^{1-3}$ Here $C^{*}$ is the critical concentration at which polymer coils begin to overlap with each other and is defined by

$$
C^{*}=\frac{3 M}{4 \pi\left\langle s^{2}\right\rangle^{3 / 2} N_{\mathrm{A}}}
$$

where $\left\langle s^{2}\right\rangle$ is the mean square radius of gyration of the polymer, $M$, the molecular weight, and $N_{\mathrm{A}}$, Avogadro's number.

In dilute solutions, the osmotic pressure is represented by the virial expansion form

$$
\Pi / C=R T\left(1 / M+A_{2} C+A_{3} C^{2}+\cdots\right)
$$

where $A_{2}$ and $A_{3}$ are the second and third virial coefficients and are expressed by

$$
A_{2}=\frac{4 \pi^{3 / 2} N_{\mathrm{A}} \Psi\left\langle s^{2}\right\rangle^{3 / 2}}{M^{2}}
$$

and

$$
A_{3}=\gamma A_{2}^{2} M
$$

It has been shown ${ }^{4,5}$ that the interpenetration function $\Psi$ is almost constant and the polymer coil behaves as a hard sphere when the molecular weight is high and the solvent is good. Moreover, $\gamma$ may be constant if the hard sphere model is also valid for $A_{3}{ }^{1,6}$ By substituting eq 1,3 , and 4 into eq 2 , we obtain

$$
\begin{aligned}
\Pi M / C R T=1 & +3 \pi^{1 / 2} \Psi\left(C / C^{*}\right) \\
& +9 \pi \Psi^{2} \gamma\left(C / C^{*}\right)^{2}+\cdots
\end{aligned}
$$

In semidilute solutions of linear polymers, the concentration dependence of the reduced osmotic pressure $\Pi M / C R T$ obeys the scaling theory of de Cloizeaux, ${ }^{6,7}$ yielding

$$
\Pi M / C R T=K_{\pi}\left(C / C^{*}\right)^{1 /(3 v-1)}
$$

where $K_{\pi}$ is a numerical constant and $v$, the excluded volume exponent in the radius of gyration $v s$. the molecular weight relationship

$$
\left\langle s^{2}\right\rangle \propto M^{2 v}
$$

The apparent second virial coefficient $S$ defined by the following equation is often used for a more detailed discussion of semidilute solutions $\mathbf{s}^{1,8}$

$$
R T S=\left[\Pi / C-\left(R T / M_{n}\right)\right] / C
$$

From eq 3, 5, and 6, we have

$$
\begin{aligned}
S / A_{2}=1 & +3 \pi^{1 / 2} \Psi \gamma\left(C / C^{*}\right) \\
& +\cdots \text { for }\left(C<C^{*}\right)
\end{aligned}
$$




$$
\begin{gathered}
S / A_{2}=K_{\mathrm{s}} \Psi^{-1}\left(C / C^{*}\right)^{(2-3 v) /(3 v-1)} \\
\text { for }\left(C>C^{*}\right)
\end{gathered}
$$

where $K_{\mathrm{s}}$ is also a numerical constant.

Therefore, the reduced osmotic pressure $\Pi M /$ $C R T$ or the reduced apparent second virial coefficient $S / A_{2}$ is a function of $C / C^{*}$ in both dilute and semidilute solutions. In concentrated solutions, however, the osmotic pressure may be explained by the theory of Flory and Huggins; ${ }^{9}$ the reduced osmotic pressure cannot be expressed as a function of $C / C^{*}{ }^{3}$

In this work, measurement of the osmotic pressure of branched polymers in a good solvent (toluene) was carried out over a wide range of polymer concentration. Since the segment density in branched polymer coils is higher than in the corresponding linear polymers of the same molecular weight, the concentration dependences of the osmotic pressure of branched and linear polymers may differ. The conformation of star-shaped polymers in semidilute solutions was discussed by Daoud and Cotton, ${ }^{10}$ taking into account two characreristic lengths $\xi$ and $\chi ; \xi$ is similar to the screening length for semidilute solutions of linear polymers and $\chi$ is the radius which is not penetrated by the branches of other molecules. The osmotic pressure of branched polymers in semidilute solutions can be well explained by the concepts presented by these authors.

\section{EXPERIMENTAL}

\section{Materials}

The samples used here were two star-shaped poly( $\alpha$-methylstyrene)s having three branches (S-22, S26) and two comb-shaped polystyrenes (KIII-F89, KI-F3) with $15-16$ branches. These samples were prepared by Fujimoto et al. in a previous work. ${ }^{11,12}$ The molecular characteristics of these samples are listed in Table I. These two comb-shaped polymers may be considered as stars rather than as combs. There is no practical difference between the branching indices calculated for these combs and for stars with the same number of branches. ${ }^{13}$ The molecular weight distriburion of branches in each sample was narrow. Each comb-shaped polymer had a distribution corresponding to the degree of branching; the samples, however, were carefully fractionated to make the distribution even narrower. ${ }^{13}$

Linear polystyrenes of narrow molecular weight distribution of Pressure Chemical Co. were used for comparison. The weight average molecular weights in Table I are from a previous paper, ${ }^{14}$ and the radii of gyration were estimated using the $\left\langle s^{2}\right\rangle$ vs. $M$ relationship $\left\langle s^{2}\right\rangle=1.38 \times 10^{-18} M^{1.19}$ determined by the least-squares method from literature data. ${ }^{14-18}$

Toluene, a good solvent, was purified by the same procedure described previously. ${ }^{4}$

\section{Osmotic Pressure Measurements}

Osmotic pressures were measured at $25^{\circ} \mathrm{C}$ using a high speed membrane osmometer of HewlettPackard Co., Ltd. When the pressure was high, a mercury or water manometer was employed to balance the pressure. The membrane used was No. 0-8 of Scheicher \& Schüll Co., Ltd., for nonaqueous solutions.

\section{Light Scattering Measurements}

Light scattering measurements for estimating molecular weight and radius of gyration were carried out at $25^{\circ} \mathrm{C}$ using a FICA-50 automatic light scattering photometer. The same experimental procedure reported previously ${ }^{4}$ was used.

Light scattering data were analyzed by the square-root plot, which utilizes the following relations:

$$
\begin{aligned}
\left(K C / R_{\theta}\right)_{\theta=0}^{1 / 2}= & \left(1 / M_{w}\right)^{1 / 2}\left(1+A_{2} M_{w} C+\cdots\right)(11-\mathrm{a}) \\
\left(K C / R_{\theta}\right)_{C=0}^{1 / 2}= & \left(1 / M_{w}\right)^{1 / 2} \\
& \times\left(1+\frac{1}{3}\left\langle s^{2}\right\rangle \frac{4 \pi}{\lambda} \sin ^{2}(\theta / 2)+\cdots\right)
\end{aligned}
$$

where $K$ is the optical constant for light scattering, $R_{\theta}$, the Rayleigh ratio at a scattering angle of $\theta, M_{w}$, the weight average molecular weight, $\lambda$, the wave length in the solution and $C$, the polymer concentration. The subscript $\theta=0$ and $C=0$ indicate the limits of the zero scattering angle and zero concentration, respectively.

\section{RESULTS}

The weight average molecular weight $M_{w}$, radius of gyration $\left\langle s^{2}\right\rangle^{1 / 2}$ and the second virial co- 
Semidilute Solutions of Branched Polymers

Table I. Sample characteristics

\begin{tabular}{|c|c|c|c|c|c|c|c|c|c|c|}
\hline \multirow{2}{*}{ Sample code } & \multirow{2}{*}{$\frac{M_{n}}{\times 10^{5}}$} & \multirow{2}{*}{$\frac{M_{w}}{\times 10^{5}}$} & \multirow{2}{*}{$f^{\mathrm{b}}$} & \multirow{2}{*}{$\frac{\left\langle s^{2}\right\rangle / \mathrm{cm}^{-2}}{\times 10^{-11}}$} & \multirow{2}{*}{$\frac{C^{*} / \mathrm{g}^{-1} \mathrm{~cm}^{3}}{\times 10^{-2}}$} & \multirow{2}{*}{$\begin{array}{r}A_{2}(\mathrm{LS}) \\
\times 10^{-4}\end{array}$} & \multirow{2}{*}{$\begin{array}{l}A_{2}(\mathrm{OS}) \\
\times 10^{-4}\end{array}$} & \multicolumn{2}{|c|}{$\psi^{\mathrm{c}}$} & \multirow{2}{*}{$g^{\prime}$} \\
\hline & & & & & & & & LS & OS & \\
\hline $\mathrm{S}-22$ & - & 9.0 & 3 & $1.4_{7}$ & $0.63_{3}$ & $2.0_{5}$ & $2.0_{9}$ & $0.2_{5}$ & $0.2_{6}$ & $0.9_{0}$ \\
\hline S-26 & - & 36.0 & 3 & $6.3_{7}$ & $0.28_{1}$ & $1.6_{5}$ & - & $0.2_{6}$ & - & $0.7_{7}$ \\
\hline KI-F3 $3^{\mathrm{a}}$ & - & 9.6 & 16 & $0.8_{5}$ & $1.5_{4}$ & $2.0_{4}$ & $2.2_{7}$ & $0.5_{6}$ & $0.6_{3}$ & $0.3_{9}$ \\
\hline KIII-F89a & - & 26.5 & 15 & $2.3_{5}$ & $0.92_{2}$ & $1.3_{5}$ & $1.5_{4}$ & $0.5_{3}$ & $0.6_{5}$ & $0.4_{7}$ \\
\hline PS-1 & 0.51 & - & - & $0.05_{5}$ & $4.9_{3}$ & - & $6.3_{6}$ & - & $0.3_{0}$ & - \\
\hline PS-2 & 1.57 & - & - & $0.21_{1}$ & $2.0_{4}$ & - & $4.6_{6}$ & - & $0.2_{8}$ & - \\
\hline PS-3 & - & $9.0_{1}$ & - & $1.6_{8}$ & $0.51_{7}$ & - & $2.9_{1}$ & - & $0.2_{6}$ & - \\
\hline PS-4 & - & $19 \cdot 3$ & - & $4.1_{7}$ & $0.28_{4}$ & - & $\left(2.5_{5}\right)$ & - & $\left(0.2_{6}\right)$ & - \\
\hline
\end{tabular}

a The number average molecular weights of a backbone $\left(M_{n 0}\right)$ and branch $\left(M_{n \mathrm{~b}}\right)$ are $9.8 \times 10^{4}$ and $17.6 \times 10^{4}$ for KIIIF89, and $5.7 \times 10^{4}$ and $5.5 \times 10^{4}$ for KI-F3, respectively.

b $f=\left(M_{w}-M_{n 0}\right) / M_{n \mathrm{~b}}$.

c LS and OS denote the $\psi$ values calculated from $A_{2}(\mathrm{LS})$ and $A_{2}(\mathrm{OS})$, respectively.

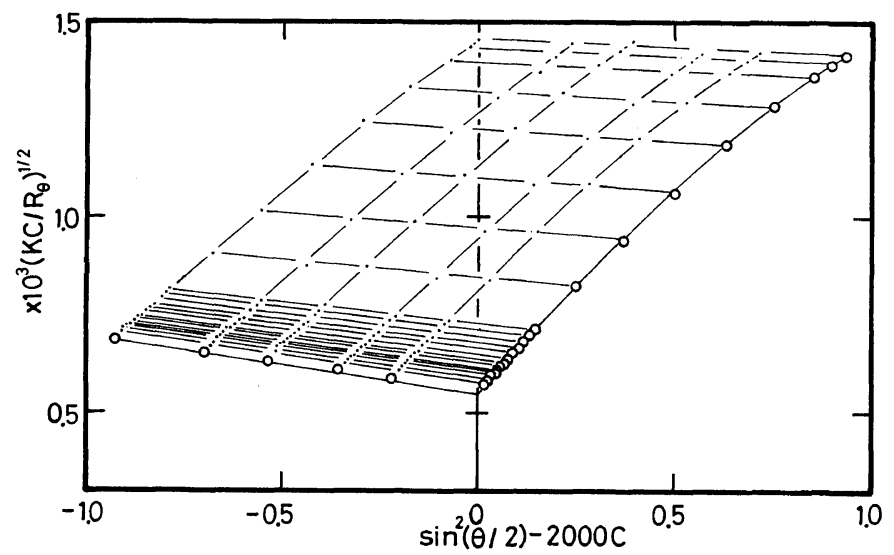

Figure 1. A Zimm plot for star-shaped poly( $\alpha$-methylstyrene) S-26 in toluene at $25^{\circ} \mathrm{C}$.

efficient $A_{2}$ (LS) for the samples studied are listed in Table I. The square-root plot is illustrated in Figure 1. The critical concentration $C^{*}$ in Table I was calculated from eq 1 using the data of $\left\langle s^{2}\right\rangle$ and $M_{n}$ or $M_{w}$. The values of $A_{2}(\mathrm{OS})$ were also evaluated from the square-root plots of osmotic pressure. To facilitate the determination of $A_{2}$ (OS) by square-root plots for certain samples (S-22, KIII-F89, KI-F3 and PS-3) the number average molecular weights were assumed to be equal to the weight average molecular weights determined by light scattering. The value of $A_{2}(\mathrm{OS})$ for PS-4 was extrapolated from the experimental relationship between $A_{2}(\mathrm{OS})$ and $M$. The $A_{2}(\mathrm{OS})$ values thus estimated and listed in Table I were used to analyze osmotic pressure data. The interpenetration function $\Psi$ in Table I was calculated by inserting the experimental values of $A_{2}, M$ and $\left\langle s^{2}\right\rangle$ into eq 3. $\Psi$ is almost independent of molecular weight in each type of sample.

All osmotic pressure data are summarized in Table II. Figure 2 shows double logarithmic plots of $\Pi / C$ vs. $C$ for star-shaped poly( $\alpha$-methylstyrene)s. The data for the corresponding linear polymers ${ }^{1}$ with high molecular weights are shown by a broken line for comparison. Figure 3 shows double logarithmic plots of $\Pi / C v s$. $C$ for comb-shaped polystyrenes, along with the data for linear polystyrenes. From Figures 2 and 3, it can be seen that osmotic pressures for branched polymers are inde- 
Table II. Osmotic pressure data for star-shaped poly( $\alpha$-methylstyrene)s, linear and comb-shaped polystyrenes in toluene at $25^{\circ} \mathrm{C}$

\begin{tabular}{|c|c|c|c|c|c|}
\hline$\times 10^{2} C$ & $\Pi$ & $\times 10^{2} \mathrm{C}$ & $\Pi$ & $\times 10^{2} \mathrm{C}$ & $\Pi$ \\
\hline $\mathrm{g} \mathrm{cm}^{-3}$ & $\mathrm{~g} \mathrm{~cm}^{-2}$ & $\mathrm{~g} \mathrm{~cm}^{-3}$ & $\mathrm{~g} \mathrm{~cm}^{-2}$ & $\mathrm{~g} \mathrm{~cm}^{-3}$ & $\mathrm{gcm}^{-2}$ \\
\hline \multicolumn{6}{|c|}{$-\mathrm{S}-22-$} \\
\hline $0.098_{4}$ & $0.04_{8}$ & $0.591_{8}$ & $0.44_{6}$ & $1.484_{9}$ & $2.39_{2}$ \\
\hline $0.198_{6}$ & $0.07_{8}$ & $0.732_{5}$ & $0.72_{3}$ & $1.835_{8}$ & $4.15_{4}$ \\
\hline $0.207_{3}$ & $0.06_{8}$ & $0.757_{7}$ & $0.64_{1}$ & $2.869_{6}$ & 10.11 \\
\hline $0.260_{2}$ & $0.10_{0}$ & $0.998_{1}$ & $1.28_{8}$ & $5.434_{1}$ & 52.13 \\
\hline $0.390_{3}$ & $0.19_{3}$ & $1.018_{3}$ & $1.10_{0}$ & & \\
\hline $0.490_{1}$ & $0.29_{5}$ & $1.320_{0}$ & $2.45_{5}$ & & \\
\hline \multicolumn{6}{|c|}{$-\mathrm{S}-26$} \\
\hline $0.517_{5}$ & $0.18_{1}$ & $1.203_{9}$ & $1.43_{7}$ & $2.132_{3}$ & $5.37_{1}$ \\
\hline $0.650_{6}$ & $0.36_{2}$ & $1.409_{1}$ & $2.05_{7}$ & $2.384_{6}$ & $6.82_{5}$ \\
\hline $0.740_{0}$ & $0.44_{8}$ & $1.606_{4}$ & $2.79_{7}$ & $2.587_{9}$ & $8.04_{8}$ \\
\hline $0.851_{4}$ & $0.60_{3}$ & $1.803_{3}$ & $3.67_{2}$ & $2.928_{4}$ & 11.14 \\
\hline $0.984_{0}$ & $0.86_{9}$ & $2.010_{1}$ & $4.83_{6}$ & & \\
\hline \multicolumn{6}{|c|}{-KIII-F89- } \\
\hline $0.518_{4}$ & $0.17_{8}$ & $1.781_{1}$ & $2.85_{3}$ & 5.575 & 56.14 \\
\hline $0.802_{2}$ & $0.50_{4}$ & $2.200_{7}$ & $5.81_{0}$ & 6.559 & 84.56 \\
\hline $0.901_{3}$ & $0.69_{4}$ & $2.507_{0}$ & $7.89_{5}$ & 7.407 & $118.5_{9}$ \\
\hline $1.007_{0}$ & $0.86_{4}$ & $2.706_{7}$ & $9.87_{9}$ & 8.361 & $152.3_{4}$ \\
\hline $1.152_{3}$ & $1.51_{0}$ & $2.911_{0}$ & $11.87_{7}$ & 9.666 & $215.1_{7}$ \\
\hline $1.312_{9}$ & $1.69_{4}$ & 3.381 & $16.51_{6}$ & 11.013 & 296.8 \\
\hline $1.545_{0}$ & $2.13_{2}$ & 4.568 & 37.26 & 11.983 & $368.4_{0}$ \\
\hline \multicolumn{6}{|c|}{$-\mathrm{KI}-\mathrm{F} 3-$} \\
\hline $0.268_{8}$ & $0.12_{9}$ & $1.345_{0}$ & $1.91_{0}$ & 5.183 & 51.16 \\
\hline $0.290_{6}$ & $0.13_{9}$ & $1.435_{4}$ & $2.18_{2}$ & 5.979 & 74.13 \\
\hline $0.419_{0}$ & $0.23_{5}$ & $1.470_{0}$ & $2.73_{4}$ & 6.427 & $130.2_{1}$ \\
\hline $0.427_{3}$ & $0.23_{9}$ & $1.688_{8}$ & $3.29_{3}$ & 6.884 & $104.6_{4}$ \\
\hline $0.529_{7}$ & $0.35_{0}$ & $1.888_{3}$ & $4.32_{4}$ & 7.354 & $123.0_{3}$ \\
\hline $0.632_{8}$ & $0.53_{8}$ & $2.089_{5}$ & $5.53_{7}$ & 8.626 & $221.9_{5}$ \\
\hline $0.632_{8}$ & $0.56_{8}$ & $2.185_{0}$ & $4.70_{9}$ & 8.769 & $184.1_{4}$ \\
\hline $0.68_{3}$ & $0.59_{4}$ & $2.384_{7}$ & $7.66_{2}$ & 10.015 & $257.9_{9}$ \\
\hline $0.802_{5}$ & $0.79_{4}$ & 2.614 & 11.27 & 10.447 & $309.7_{5}$ \\
\hline $0.979_{7}$ & $0.98_{0}$ & 2.933 & 13.35 & 10.881 & $330.0_{2}$ \\
\hline $1.021_{1}$ & $1.04_{9}$ & 3.102 & 15.14 & 12.112 & $426.5_{9}$ \\
\hline $1.021_{1}$ & $1.10_{1}$ & 3.562 & 25.15 & 13.103 & $522.4_{2}$ \\
\hline $1.061_{0}$ & $1.33_{7}$ & 3.809 & 22.92 & 13.188 & $516.8_{4}$ \\
\hline $1.085_{2}$ & $1.33_{8}$ & 4.575 & 35.51 & 14.504 & $658.1_{9}$ \\
\hline $1.241_{6}$ & $1.51_{5}$ & 4.787 & 60.03 & & \\
\hline
\end{tabular}

pendent of molecular weight and agree with those for the corresponding linear polymers at high polymer concentrations.

The apparent second virial coefficients $S$ of two types of branched polymers and linear polystyrenes are plotted against polymer concentration in Figures 4 and 5.
Table II. (continued) $\operatorname{poly}(\alpha$-methylstyrene)s, linear and comb-shaped polystyrenes in toluene at $25^{\circ} \mathrm{C}$

\begin{tabular}{|c|c|c|c|c|c|}
\hline$\times 10^{2} \mathrm{C}$ & $\Pi$ & $\times 10^{2} \mathrm{C}$ & $\Pi$ & $\times 10^{2} \mathrm{C}$ & $\Pi$ \\
\hline $\mathrm{g} \mathrm{cm}^{-3}$ & $\mathrm{~g} \mathrm{~cm}^{-2}$ & $\mathrm{~g} \mathrm{~cm}^{-3}$ & $\mathrm{~g} \mathrm{~cm}^{-2}$ & $\mathrm{~g} \mathrm{~cm}^{-3}$ & $\mathrm{~g} \mathrm{~cm}^{-2}$ \\
\hline \multicolumn{6}{|c|}{-PS-1- } \\
\hline $0.082_{6}$ & $0.43_{2}$ & $1.151_{5}$ & $8.14_{1}$ & $5.679_{6}$ & 105.8 \\
\hline $0.213_{5}$ & $1.13_{8}$ & $1.328_{4}$ & $9.87_{0}$ & $8.819_{9}$ & 246.4 \\
\hline $0.338_{9}$ & $1.87_{8}^{\circ}$ & $2.365_{6}$ & 22.82 & $10.981_{5}$ & 400.8 \\
\hline $0.589_{3}$ & $3.53_{0}$ & $3.323_{3}$ & 39.14 & $12.889_{7}$ & 585.0 \\
\hline $0.843_{1}$ & $5.44_{6}$ & $4.138_{0}$ & 60.29 & & \\
\hline \multicolumn{6}{|c|}{-PS-2- } \\
\hline $0.171_{8}$ & $0.25_{9}$ & $0.803_{2}$ & $2.09_{6}$ & $4.671_{2}$ & 51.75 \\
\hline $0.257_{8}^{\circ}$ & $0.44_{1}$ & $0.966_{7}$ & $2.79_{4}$ & $6.166_{7}$ & 95.15 \\
\hline $0.313_{0}^{\circ}$ & $0.63_{5}$ & $1.232_{7}$ & $2.88_{5}$ & $7.914_{5}$ & 167.7 \\
\hline $0.409_{1}$ & $0.81_{4}$ & $1.533_{7}$ & $6.01_{2}$ & $10.895_{4}$ & 345.6 \\
\hline $0.482_{5}$ & $1.04_{7}$ & $1.970_{3}$ & $9.33_{9}$ & $13.751_{3}$ & 630.4 \\
\hline $0.579_{9}$ & $1.29_{3}$ & $2.668_{8}$ & 16.57 & & \\
\hline $0.669_{3}$ & $1.63_{3}$ & $3.058_{2}$ & 21.83 & & \\
\hline \multicolumn{6}{|c|}{-PS-3- } \\
\hline $0.237_{7}$ & $0.08_{7}$ & $0.872_{4}$ & $1.17_{8}$ & 4.442 & 40.33 \\
\hline $0.323_{3}$ & $0.17_{7}$ & $1.133_{0}$ & $1.92_{6}^{\circ}$ & 6.065 & 84.60 \\
\hline $0.436_{1}$ & $0.27_{7}$ & 1.244 & $2.40_{1}$ & 7.596 & 146.6 \\
\hline $0.513_{2}$ & $0.42_{4}$ & 1.612 & $4.22_{3}$ & 8.584 & 198.8 \\
\hline $0.633_{8}$ & $0.64_{0}$ & 2.250 & $8.70_{8}$ & 11.158 & 368.2 \\
\hline $0.739_{9}^{\circ}$ & $0.84_{3}$ & 2.575 & $11.84^{\circ}$ & 13.878 & 690.1 \\
\hline \multicolumn{6}{|c|}{-PS-4- } \\
\hline $0.250_{5}$ & $0.09_{5}$ & $0.951_{5}$ & $1.18_{9}$ & 3.800 & 27.20 \\
\hline $0.358_{9}$ & $0.20_{7}$ & $1.296_{9}$ & $2.45_{1}$ & 3.975 & 30.29 \\
\hline $0.420_{7}$ & $0.26_{8}$ & $1.727_{3}$ & $4.62_{9}$ & 4.766 & 48.99 \\
\hline $0.574_{4}$ & $0.45_{4}$ & 2.259 & $8.72_{0}$ & 7.643 & 147.8 \\
\hline $0.764_{0}$ & $0.81_{0}$ & 2.993 & 16.19 & & \\
\hline
\end{tabular}

\section{DISCUSSION}

As evident from Table I, the interpenetration function $\Psi$ depends on the degree of branching but appears to be independent of the chain length of branches provided the molecular weight is high. Thus, it may be expected that the reduced osmotic pressure $\Pi M / C R T$ of each group of branched polymer in dilute solutions can be expressed as a function of $C / C^{*}$.

In semidilute solutions $\left(C>C^{*}\right)$, linear polymer coils in a good solvent overlap with each other to form a uniform network structure. Inside this network, the blobs behave as independent polymer coils of size $\xi$ which depends only on polymer concentration according to ${ }^{2}$ 


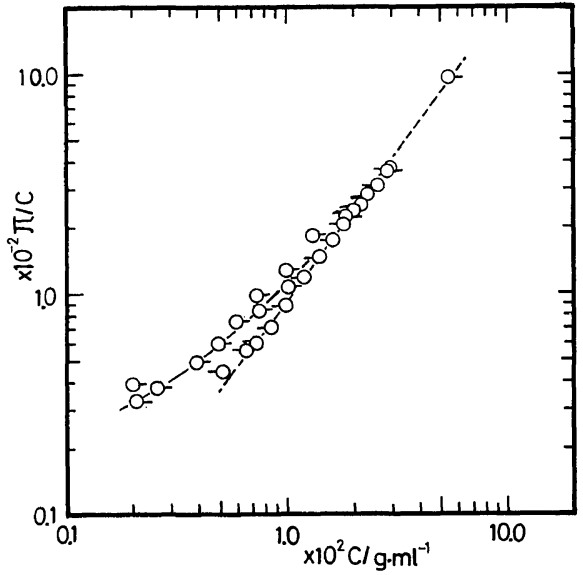

Figure 2. Osmotic pressure data of star-shaped poly $(\alpha-$ methylstyrene)s in toluene at $25^{\circ} \mathrm{C}$. The symbols $\bigcirc$ - and $-\mathrm{O}$ denote the data for S-22 and S-26, respectively. The broken line shows the data for linear $\operatorname{poly}(\alpha-$ methylstyrene)s. ${ }^{1}$

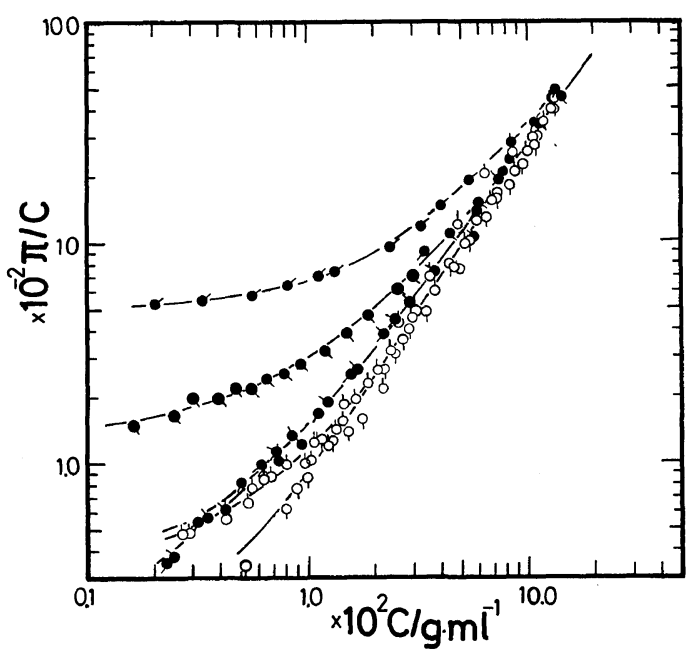

Figure 3. Osmotic pressure data of comb-shaped polystyrenes in toluene at $25^{\circ} \mathrm{C}$. The symbols $Q$ and $\checkmark$ denote the data for KIII-F89 and KI-F3, respectively. The symbols $\boldsymbol{\gamma}, \mathbf{Q}, \boldsymbol{\rho}$, and $\boldsymbol{\gamma}$ denote the data of linear polystyrenes, PS-1, PS-2, PS-3, and PS-4, respectively.

$$
\xi \propto C^{-v /(3 v-1)}
$$

Since osmotic pressure is proportional to $1 / \xi^{3},{ }^{2}$ $\Pi M / C R T$ of linear polymers in semidilute solutions are proportional to $\left(C / C^{*}\right)^{1 /(3 v-1)}$, as expressed by eq 6 .

If $v$ in eq 7 for branched polymers is the same as

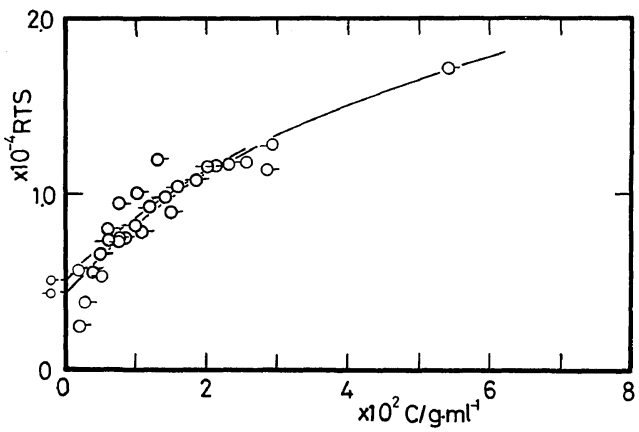

Figure 4. Apparent second virial coefficients of starshaped poly $\left(\alpha\right.$-methylstyrene)s in toluene at $25^{\circ} \mathrm{C}$. The symbols are the same as in Figure 2. The symbols of intercepts at $C=0$ denote the second virial coefficients determined by light scattering $A_{2}$ (LS).

that for linear polymers and $\xi$ for branched polymers has the same concentration dependence as eq 12 for linear polymers, $\Pi M / C R T$ for branched polymers is expressed by

$$
\Pi M / C R T=K_{\pi} g^{\prime-3 / 2(3 v-1)}\left(C / C^{*}\right)^{1 /(3 v-1)}
$$

where $K_{\pi}$ is the proportionality constant in eq 6 for linear polymers, $g^{\prime}$, the mean square radius of gyration of a branched polymer $\left\langle s^{2}\right\rangle_{b}$ relative to that of the linear polymer having the same molecular weight $\left\langle s^{2}\right\rangle_{1}$, i.e., $g^{\prime}=\left\langle s^{2}\right\rangle_{b} /\left\langle s^{2}\right\rangle_{1}$, and $C^{*}$ is given by eq 1 with $\left\langle s^{2}\right\rangle_{b}$ for $\left\langle s^{2}\right\rangle$. Equation 13 may apply not only to star-shaped polymers but also to comb-shaped polymers at high degrees of coil overlapping, since osmotic pressures for branched polymers agree with those of the corresponding linear polymers at least at high concentrations, irrespective of molecular weight and degree of branching (see Figures 2 and 3), and also since $g^{\prime}$ depends on the degree of branching but appears almost independent of molecular weight, as shown in Table I.

If the degree of coil overlapping is not too high in semidilute solutions of highly branched polymers, the network structure cannot be as uniform as in linear polymer solutions. In the vicinity of a branch point, there may be a region of radius $r_{\mathrm{c}}$, from which the segments of other molecules are excluded due to the high segment density of branched polymers, as was assumed by Daoud and Cotton. ${ }^{10}$ Assuming that the osmotic pressure of a branched polymer in a semidilute solution is proportional to the number of contact points outside $r_{\mathrm{c}}$, we obtain 


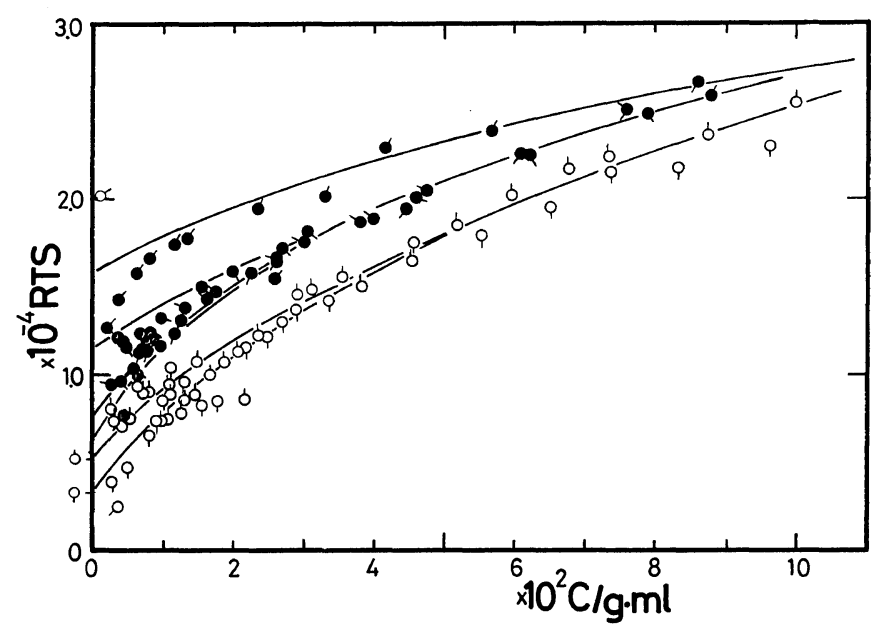

Figure 5. Apparent second virial coefficients of comb-shaped polystyrenes in toluene at $25^{\circ} \mathrm{C}$. The symbols are the same as in Figure 3. The symbols of the intercepts at $C=0$ denote the second virial coefficients determined by light scattering $A_{2}$ (LS).

$$
\Pi / R T \propto F / \xi^{3}
$$

where $F$ is the fraction of polymer segments outside $r_{\mathrm{c}}$. By taking into account the factor $F$ eq 13 gives

$$
\Pi M / C R T=K_{\pi} g^{\prime-3 / 2(3 v-1)}\left(C / C^{*}\right)^{1 /(3 v-1)} F
$$

and, similarly,

$$
S / A_{2}=K_{\mathrm{s}} \Psi^{-1} g^{-3 / 2(3 v-1)}\left(C / C^{*}\right)^{(2-3 v) /(3 v-1)} F
$$

Thus, the effect of branches becomes apparent through the $g^{\prime}$ and $F$ factors.

To calculate $F$, we consider a star-shaped polymer with $f$ branches each having $n$ segments. In a $\theta$-solvent, the probability of finding the $n$-th segment at a distance $r$ from a branch point is given by

$$
P_{n}(r)=\left(3 / 2 \pi\left\langle r^{2}\right\rangle\right)^{3 / 2} \exp \left(-3 r^{2} / 2\left\langle r^{2}\right\rangle\right)
$$

where $\left\langle r^{2}\right\rangle=a^{2} n$ and $a$ is the segment length. We assume that the expansion factor $\alpha$ of a star-shaped polymer of $f$ branches having $n$ segments is given by the theory of Candau, Rempp and Benoit, ${ }^{19}$ when neglected the triple contact term and derived

$$
\alpha^{5} \propto(f n)^{1 / 2} g^{-3 / 2}
$$

where $g$ is the unperturbed radius of gyration of the branched polymer relative to that of the corresponding linear polymer $\left(g=\left\langle s^{2}\right\rangle_{b 0} /\left\langle s^{2}\right\rangle_{10}\right)$. We further assume that the expansion factor for $\left\langle r^{2}\right\rangle$ in a good solvent is also given by eq 18 . We then obtain

$$
\left\langle r^{2}\right\rangle=a^{2} n \alpha^{2} \propto a^{2} n(n f)^{1 / 5} g^{-3 / 5}
$$

Subsitution of eq 19 into eq 17 gives an approximate distribution of the $n$-th segment in a branch in a good solvent. If we integrate the distribution function for $n$ over all segments, assuming $r^{2} \ll a^{2} N$ and multiply the result by $f$, we obtain the segment density $C(r)$ as a function of the distance $r$ from the branch point

$$
C(r) \propto g^{1 / 2} f^{5 / 6} a^{-5 / 3} r^{-4 / 3}
$$

If the sements of other molecules cannot enter the region of radius $r_{\mathrm{c}}, C(r)$ is equal to the average concentration $C$ of segments in the solution at $r=r_{\mathrm{c}}$. Thus, we have

$$
r_{\mathrm{c}} \propto f^{5 / 8} g^{3 / 8} a^{-5 / 4} C^{-3 / 4}
$$

If the number of segments inside $r_{\mathrm{c}}$ in a branch is denoted by $n_{\mathrm{c}}$, the fraction of segments outside $r_{\mathrm{c}}, F$, is

$$
F=1-n_{\mathrm{c}} / N
$$

Since the ratio $n_{\mathrm{c}} / N$ is proportional to $\left(r_{\mathrm{c}} /\left\langle R^{2}\right\rangle^{1 / 2}\right)^{1 / 0.6}$ where $\left\langle R^{2}\right\rangle$ is the mean square end-to-end distance of a branch, $F$ is given by

$$
F=1-\left(r_{\mathrm{c}} /\left\langle r^{2}\right\rangle^{1 / 2}\right)^{1 / 0.6}=1-K^{\prime}\left(C / C^{*}\right)^{-1.25}
$$

where $K^{\prime}$ is a numerical constant. Here, it should be noted that we can derive the same equation as eq 23 


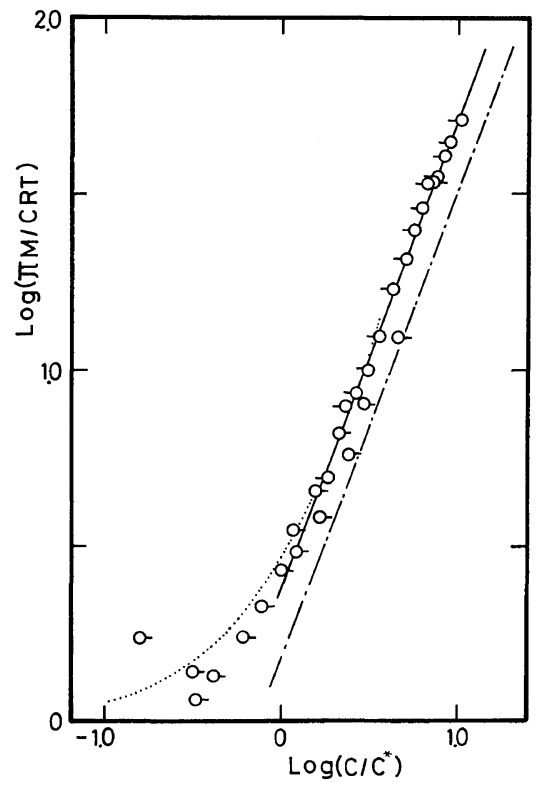

Figure 6. Double logarithmic plot of reduced osmotic pressure $\Pi M / C R T$ vs. $C / C^{*}$ for star-shaped polymers. The symbols are the same as in Figure 2. The chain line denotes the data for linear polymers. ${ }^{1}$ The solid line denotes eq 15 , assuming $v=0.58_{5},{ }^{1} g^{\prime}=0.85, K^{\prime}=0$, and $K_{\pi}=1.5 .^{1}$ The dotted curve denotes eq 5 assuming $\psi=0.26$ and $\gamma=0.25$.

from the theory of Daoud and Cotton ${ }^{10}$ for a starshaped polymer with long branches, although their equation for $C(r)$ is different from eq 20. If, more generally, $v$ is used in place of 0.6 in eq $23, F$ is written as

$$
F=1-K^{\prime}\left(C / C^{*}\right)^{-1 /(3 v-1)}
$$

Since $F$ is a function of $C / C^{*}, \Pi M / C R T$ and $S / A_{2}$ for branched polymers should be functions of $C / C^{*}$. As the polymer concentration increases, $F$ should approach unity.

Figures 6 and 7 show the double logarithmic plots of $\Pi M / C R T$ vs. $C / C^{*}$ for star-shaped and combshaped polymers, respectively. It appears that the reduced osmotic pressure $\Pi M / C R T$ for both starshaped and comb-shaped polymers can be expressed as a function of $C / C^{*}$. The data for the corresponding linear polymers ${ }^{1}$ are also shown for comparison. In these figures, the dotted lines show the values calculated from eq 5 . The calculated values on the basis of eq 15 are shown by solid lines, with $K^{\prime}$ in eq. 24 taken as an adjustable parameter.

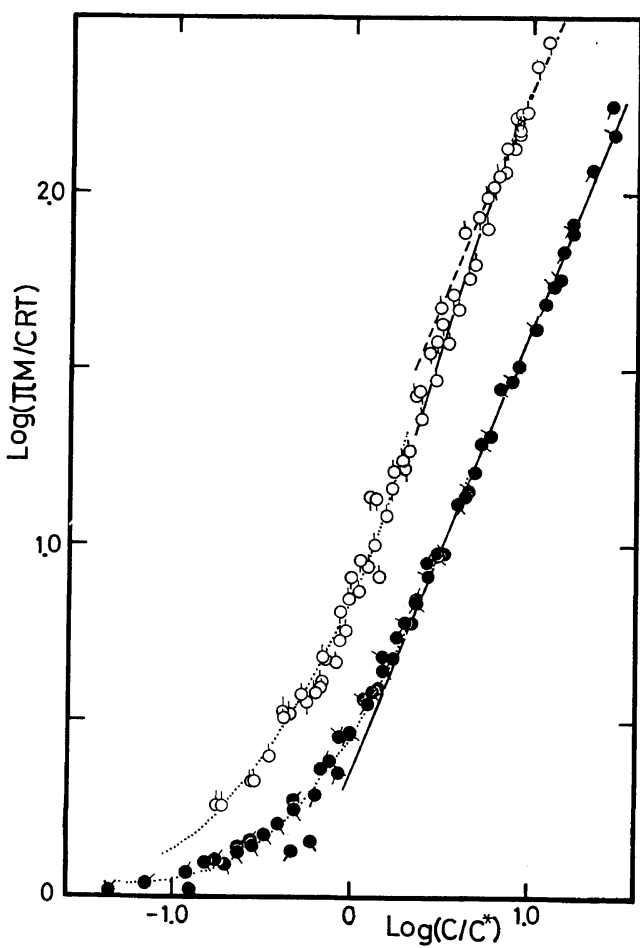

Figure 7. Double logarithmic plots of reduced osmotic pressures $\Pi M / C R T$ vs. $C / C^{*}$ for linear and combshaped polystyrenes. The symbols are the same as in Figure 3. The solid line for linear polymers denotes eq 6 , assuming $K_{\pi}=2.2$ and $v=0.59_{5}$. The solid curve for comb-shaped polystyrenes denotes eq 15 assuming $K_{\pi}=2.2, v=0.59_{5}, K^{\prime}=0.95$, and $g^{\prime}=0.42$. The broken line denotes eq 15 , assuming that $K^{\prime}=0$ and the other parameters are the same as for the solid curve. The dotted curves denote eq 5 assuming $\psi=0.63$ and $\gamma=0.25$ for the comb-shaped polymers, and $\psi=0.26$ for the linear polymers.

The values of the other parameters used for calculation are given in the figure captions. The values of $K_{\pi}$ were obtained from the corresponding linear polymers, and the values of $g^{\prime}$ determined experimentally.

In Figures 8 and 9, the experimental values of $S / A_{2}$ for star-shaped and comb-shaped polymers, respectively, are plotted against $C / C^{*}$. The values of the parameters $v, g^{\prime}, \Psi$ and $K^{\prime}$ used for calculation are the same as those in Figures 6 and 7. The values of $K_{\mathrm{s}}$ were taken from the corresponding linear polymers.

Figures 6 and 8 show that the experimental data for star-shaped polymers are in good agreement 


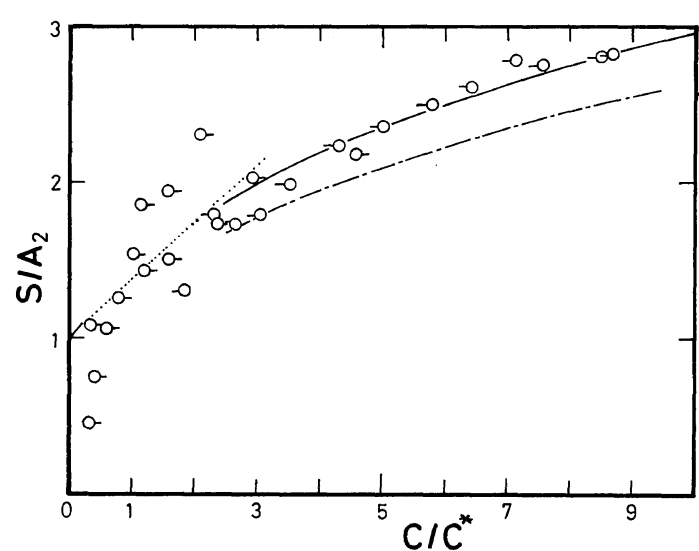

Figure 8. Plots of $S / A_{2}$ against $C / C^{*}$. The symbols are the same as in Figure 2. The solid curve denotes eq 16 assuming $K_{\mathrm{s}}=0.26$, obtained for the corresponding linear polymers. ${ }^{1}$ The chain line denotes the data for linear polymers. ${ }^{1}$ The dotted line denotes eq 9 . The parameter values used are the same as in Figure 6.

with the lines calculated with $K^{\prime}=0$, i.e., $F=1$. This indicates that, in solutions of star-shaped polymers having three branches, there is no region from which the segments of other molecules are excluded. The difference in reduced osmotic pressures of the star-shaped and corresponding linear polymers arises only from the $g^{\prime}$ factor, that is, the difference in the radii of gyration.

On the other hand, the experimental data for comb-shaped polymers agree with the values calculated with $K^{\prime}=0.95$. In both Figures 7 and 9, the values computed from eq 15 with $F=1$ agree with experimental data only at large values of $C / C^{*}$ $(>8)$. This implies that a polymer with many branches has a region into which the other molecules cannot penetrate and that this region disappears as coils increasingly overalp. The reason why $K^{\prime}=0$ is valid for star-shaped polymers with $f=3$ but $K^{\prime}=0.95$ for highly branched polymers is not clear at present. The assumption that the segment distribution in branched polymers is similar to that in linear polymers should be applied with considerable reservation to highly branched polymers. In the vicinity of a branch point in a highly branched polymer, the segment density may be higher than predicted by eq 20 . Therefore, it may be expected that parameter $K^{\prime}$ varies with the degree of branching.

As can be seen from Table I, the values of $\Psi$ for

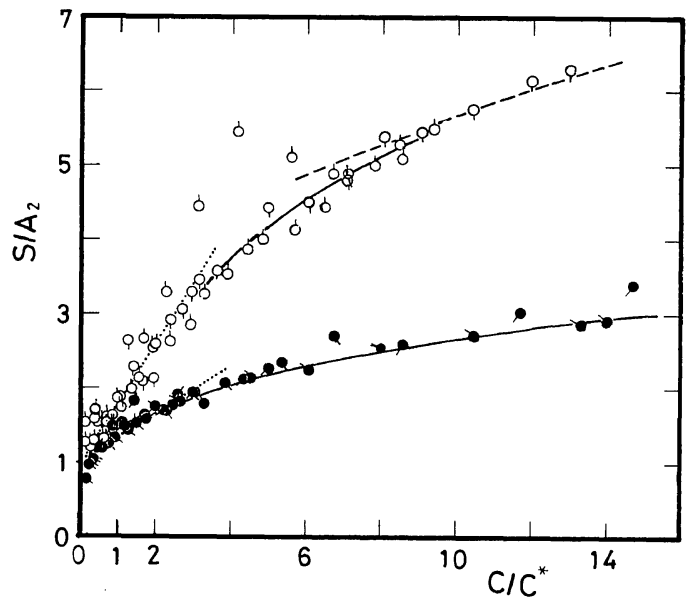

Figure 9. Plots of $S / A_{2}$ against $C / C^{*}$ for comb-shaped and linear polystyrenes. The symbols are the same as in Figure 3. The solid curves for comb-shaped polymers and for linear polystyrenes denote the calculated values of eq 16 and 10 , respectively. The value of $K_{\mathrm{s}}$ is assumed to be 0.37 for both solid lines. The values of the other parameters used are the same as in Figure 6. The broken curve denotes eq 16 , assuming $K^{\prime}=0$. The values of the other parameters are the same as in Figure 7. The dotted line denotes eq 9. The parameter values used are the same as in Figure 7.

star-shaped polymers are slightly larger than $0.2_{1}$ for linear poly( $\alpha$-methylstyrene)s, ${ }^{4,5}$ but the values of $\Psi$ for comb-shaped polymers are considerably larger than those for linear polystyrenes. ${ }^{18,20,21}$ This fact indicates that branched polymers probably behave more like hard spheres than linear polymers. This effect may persist even in semidilute solutions for highly branched polymers.

Acknowledgments. We should like to express our appreciation to Professor M. Nagasawa of Nagoya University for his many helpful suggestions and comments, and also to Mr. M. Itoh and Mr. F. Tomikawa for their assistance in making the light scattering and osmotic pressure measurements.

\section{REFERENCES}

1. I. Noda, N. Kato, T. Kitano, and M. Nagasawa, Macromolecules, 14, 668 (1981).

2. P. G. de Gennes, "Scaling Concepts in Polymer Physics," Cornell University Press, Ithaca, New York and London, 1980.

3. I. Noda, Y. Higo, N. Ueno, and T. Fujimoto, 
Macromolecules, submitted.

4. T. Kato, K. Miyaso, I. Noda, T. Fujimoto, and M. Nagasawa, Macromolecules, 3, 777 (1970).

5. I. Noda, T. Kitano, and M. Nagasawa, J. Polym. Sci., Polym. Phys. Ed., 17, 1129 (1977).

6. J. des Cloizeaux and I. Noda, Macromolecules, 15, 1505 (1982).

7. J. des Cloizeaux, J. Phys. (Paris), 36, 281 (1975).

8. M. Fixman, J. Chem. Phys., 33, 370 (1960).

9. P. J. Flory, "Principles of Polymer Chemistry," Cornell University Press, Ithaca, New York, 1953.

10. M. Daoud and J. P. Cotton, J. Phys. (Paris), 43, 531 (1982).

11. T. Fujimoto, S. Tani, K. Takano, M. Ogawa, and M. Nagasawa, Macromolecules, 11, 673 (1978).

12. T. Fujimoto, H. Nakamura, and M. Nagasawa, Macromolecules, 3, 57 (1970).

13. I. Noda, T. Horikawa, T. Kato, T. Fujimoto, and M.
Nagasawa, Macromolecules, 3, 795 (1970).

14. T. Kato, A. Itsubo, Y. Yamamoto, T. Fujimoto, and M. Nagasawa, Polym. J., 7, 123 (1975).

15. G. C. Berry, J. Chem. Phys., 44, 4550 (1966).

16. R. G. Kirste and G. Wild, Makromol. Chem., 121, 174 (1969).

17. H. Utiyama, S. Utsumi, Y. Tsunashima, and M. Kurata, Macromolecules, 11, 506 (1978).

18. I. Noda, M. Imai, T. Kitano, and M. Nagasawa, Macromolecules, 16, 425 (1983).

19. F. Candau, R. Rempp, and B. Benoit, Macromolecules, 5, 627 (1972).

20. M. Fukuda, M. Fukutomi, Y. Kato, and T. Hashimoto, J. Polym. Sci., Polym. Phys. Ed., 12, 871 (1974).

21. Y. Miyaki, Y. Einaga, and H. Fujita, Macromolecules, 11, 1180 (1978). 